\title{
Transport de la charge solide dans le bassin versant de l'Orne. Composition, variabilité et flux (1994-1995)
}

\section{Nature and transport of the suspended load in the Orne basin (1994-1995)}

\author{
A. Nasseh ${ }^{1-3}$, H. Texier ${ }^{2}$, M. Lacroix ${ }^{4}$, B. Ouddane ${ }^{5}$ \\ 1 Ecole Normale Supérieure, BP 209, Martil, Tetouan. Maroc \\ 2 Centre du CNRS. Géosciences, 24, rue du Tilleul, Unversité de Caen 14032 Caen Cedex \\ 3 Facultè des Sciences. BP 1796. Fes-Atlas. Maroc \\ 4 Laboraloire de géologie. Université de Rouen. 76134 Mont Saint Aignan Cedex \\ 5 Laboratoire de chimie analytique et marine. Universite des Sciences et Technologies de Lille, 59655 Villeneuve d'Ascq Codex
}

Resumé. - Notre étude vise à établir les bilans de l'érosion et des apports solides au système estuarien au niveau du bassin versant de l'Orne (Calvados, France) à partir d'un suivi d'amont en aval du transfert des MES effectué en onze stations réparties sur le bassin et choisies en fonction des caractéristiques lithologiques et hydrologiques du bassin. Les flux annuels des différentes phases à l'exutoire, entrant dans l'estuaire de l'Orne, sont évalués à partir d'un suivi dont la fréquence est basée sur le régime hydrique de la rivière. Les analyses portent sur les teneurs des MES en éléments majeurs ( $\mathrm{Si}, \mathrm{Al}, \mathrm{Fe}, \mathrm{Ca}, \mathrm{Mg}, \mathrm{Na}, \mathrm{K}$ ), mineurs et traces (Ti, Cd, $\mathrm{Cu}, \mathrm{Cr}, \mathrm{Pb}, \mathrm{Zn}$ et $\mathrm{Ni}$ ). L'étude menée pour la première fois sur ce bassin, a permis de déterminer les niveaux de teneurs, de décrire la variabilité saisonnière et spatiale des concentrations, d'évaluer l'influence des deux principaux affluents, Rouvre et Noireau et de calculer les flux exportés.

Cette étude a été menée dans le cadre du programme "Dynamique et Bilan de la Terre II", financé par l'institut des Sciences de l'Univers, sur le thème "Erosion et Hydrologie" ".

Mots-clés. - rivières, matières en suspension, métaux, flux.

Abstract. - This study aimed at assessing erosion and solid inputs in the estuarine system of the Orne basin (Calvados, France). Transport of suspended matter from upstream to downstream was monitored at eleven sites throughout the basin, chosen for their specific lithological and hydrological characteristics. Annual fluxes of the different phases entering the Orne estuary were estimated by regular monitoring, whose frequency was determined depending on the hydric regime of the river. Concentrations of suspended matter were analyzed for major elements ( $\mathrm{Si}, \mathrm{Al}, \mathrm{Fe}, \mathrm{Ca}, \mathrm{Mg}, \mathrm{Na}, \mathrm{K}$ ), minor and trace elements ( $\mathrm{Ti}, \mathrm{Cd}, \mathrm{Cu}, \mathrm{Cr}, \mathrm{Pb}, \mathrm{Zn}$ and $\mathrm{Ni}$ ).

This study, conducted for the first time in this basin, enabled determining the levels of 
concentrations, describing seasonal and spatial variations in these concentrations, estimating the impact of the two major affluents, the Rouvre and the Noreau, and calculating exported fluxes.

The study was carried out in the context of a broader project to examine the relationship between erosion and hydrology.

Key-words. - rivers, suspended matter, metals, fluxes.

\section{INTRODUCTION}

Les études portant sur les variations de la composition chimique des eaux naturelles et sur les processus la régissant constituent une donne nécessaire pour connaître la variabilité géographique des processus d'altération, l'influence de l'activité anthropique et les flux exportés à l'Océan Mondial: Martin \& Meybeck, 1979; Milliman \& Meade, 1983; Snoussi, 1988; Horovitz et al., 1990; Abdel-Hamid et al., 1992; Probst et al., 1992; Thomas \& Meybeck, 1992; Shankar \& Manjunatha, 1994 ; Idlafkih et al., 1995; Zhang et al., 1995; Sinawi \& Walling, 1996.

Dans le cadre du programme national français "Dynamique et Bilan de la Terre II", une étude relativement complète de la géochimie des eaux a été menée dans le bassin de l'Orne sur deux cycles complets, 1993-1994 et 1994-1995 (Nasseh et al., 1996). Nous exposons dans ce travail les résultats concernant les niveaux et la dynamique de transport des métaux majeurs et en traces particulaires, leurs variations dans le temps et dans l'espace et les bilans calculés. Ce travail constitue la première étude quantitative et qualitative des apports solides à l'estuaire de l'Orne.

\section{PRESENTATION GENERALE}

Situé au Nord-Ouest de la France, le bassin de l'Orne couvre à May-surOrne une superficie de $2680 \mathrm{~km}^{2}$ (carte). Le fleuve d'une longueur de $175 \mathrm{~km}$, prend sa source à une altitude de $218 \mathrm{~m}$ dans la campagne de Sées et se jette à Ouistreham dans la mer de la Manche. Ce bassin constitue de par la nature diversifiée du sous-sol, des formations superficielles et des écoulements, un atelier original sous climat tempéré. Deux secteurs séparés schématiquement par une diagonale Nord-Ouest/Sud-Est et nettement différenciés par leurs aspects physiques généraux se distinguent dans le bassin (Nasseh et al., 1996) :

- Un domaine silico-alumineux relativement homogène au Sud-Ouest, 
composé essentiellement de schistes, de schistes gréseux et de granites. Cette partie très arrosée (plus de $900 \mathrm{~mm}$ en moyenne) est caractérisée par de fortes pentes, une faible perméabilité, des coefficients de ruissellement élevés et une forte densité du réseau hydrographique. Elle est traversée par les principaux affluents de l'Orne dont la Rouvre et le Noireau.

- Un domaine carbonaté hétérogène au Nord-Est qui comprend les plateaux des campagnes de Caen et d'Argentan, constitué principalement de calcaire et de calcaire marneux. Les altitudes culminent à $250 \mathrm{~m}$, la lame d'eau est inférieure à $650 \mathrm{~mm}$ en moyenne, le réseau hydrographique est très lâche et les terrains perméables permettent la formation de nappes souterraines de grande étendue et de forte capacité.

Le bassin a une vocation essentiellement agricole: cultures céréalières dans les campagnes (Caen, Laize, Argentan) et élevage dans le Bocage Normand et les fonds de vallées. L'activité industrielle se cantonne principalement dans l'agglomération caennaise (sidérurgie portuaire, industrie automobile, et agro-alimentaire).

Le régime climatique est typiquement océanique dominé par des pluies fines réparties sur toute l'année. Les précipitations moyennes annuelles varient de 600 à plus de $1000 \mathrm{~mm}$.
L'hydrologie de l'Orne est influencée principalement par les affluents de la rive gauche et notamment le Noireau. Les montées de crue sont généralement brutales, leur propagation ainsi que les décrues sont rapides. La sensibilité du réseau hydrographique aux précipitations s'explique par la nature principalement imperméable des bassins versants armoricains et par leurs caractères morphométriques: indice de compacité de Gravélius $K_{c}=1,8$; rapport de confluence $\mathrm{RC}$ de l'ordre de 2 ; densité de drainage forte (Nasseh et al., 1996). Le régime hydrologique fait apparaître deux périodes nettement distinctes dans leur répartition annuelle : une période de hautes eaux (de novembre-décembre à avril; $70 \mathrm{~m}^{3} / \mathrm{s}$ en moyenne) et une période des basses eaux (de juin-juillet à octobre; $8 \mathrm{~m}^{3} / \mathrm{s}$ en moyenne). Le débit moyen annuel $\left(Q_{m a}\right)$ calculé à Maysur-Orne a atteint respectivement 33 et $39 \mathrm{~m}^{3} / \mathrm{s}$ durant les deux cycles étudiés. II est très supérieur au débit moyen interannuel de $20,5 \mathrm{~m}^{3} / \mathrm{s}$ calculé sur la période 1984-1993. En outre, l'Orne a connu en janvier-février 1995 la troisième crue du siècle qui a duré plusieurs jours et atteint $452 \mathrm{~m}^{3} / \mathrm{s}$.

\section{MÉTHODOLOGIE}

Les données utilisées dans cette étude ont été recueilles à partir d'un suivi d'amont en aval des transferts des métaux majeurs et en traces par- 
ticulaires, réalisé mensuellement de janvier 1994 à décembre 1995, en onze stations réparties sur le bassin versant, entre Argentan et Caen et choisies en fonction des caractéristiques lithologiques et hydrologiques du bassin (carte 1).

Les flux exportés sont calculés à la station N11 de May-sur-Orne à l'entrée de l'estuaire, à partir d'un suivi où le rythme des prélèvements a été adapté au régime hydrique: généralement hebdomadaire en hivernage et journalier pendant certaines crues.

Trente à cinquante litres d'eau sont recueilles à chaque station. L'eau prélevée est rapidement filtrée sur des filtres de porosité $0,45 \mu \mathrm{m}$.

La charge en MES est obtenue après filtration, par séchage puis pesée.

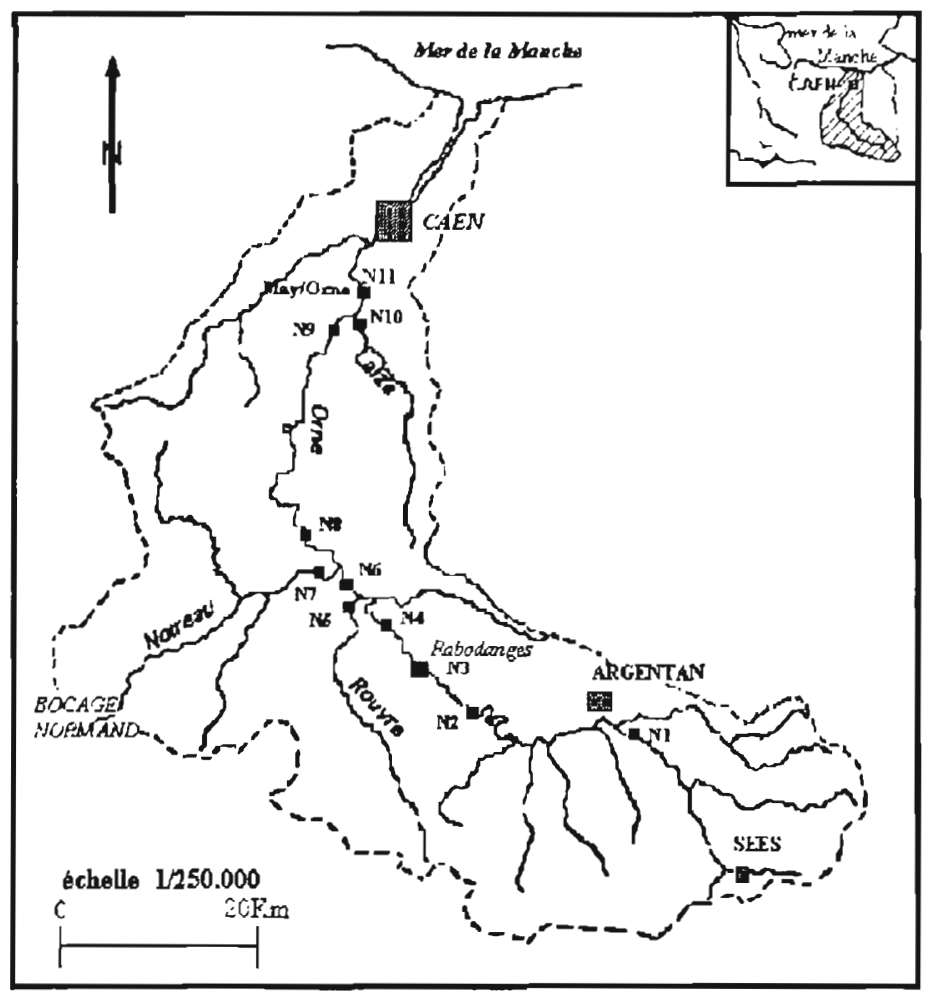

Carte 1: Bassin de l'Orne el situation des stations de prélèvements.

Plan 1: Orne Basin and situation of samples. 
L'analyse granulométrique est effectuée sur l'échantillon brut à l'aide d'un compteur de particules Coulter (COULTER TA-II à deux orifices, associé à un module échantillonneur ASP et relié à un micro-ordinateur HP-86B pour l'acquisition des données). Elle permet de caractériser les différentes fractions granulométriques contenues dans des suspensions naturelles (Dupont et al., 1994; Lafite, 1995). Une identification au microscope électronique à balayage (MEB) a été menée parallélement sur certains échantillons.

Les teneurs en carbone organique (COP) sont mesurées par combustion à haute température sur un analyseur carbone LECO CS 125. Les échantillons sont décarbonatés avant analyses avec du $\mathrm{HCl} 2 \mathrm{~N}$ durant une nuit à $60^{\circ} \mathrm{C}$. La précision est excellente pour tous les échantillons puisque les coefficients de variation sont inférieures à $6 \%$.

Les teneurs des éléments $\mathrm{Al}, \mathrm{Fe}$, $\mathrm{Ca}, \mathrm{Mg}, \mathrm{K}, \mathrm{Na}$ et $\mathrm{Ti}$ sont déterminées par spectrométrie d'émission atomique (Torche à plasma: appareil A.R.L. modèle 3510 , piloté par un ordinateur IBM-PCXT). Les analyses des métaux en traces $\mathrm{Cd}, \mathrm{Cr}, \mathrm{Cu}, \mathrm{Pb}$, $\mathrm{Zn}$ et $\mathrm{Ni}$ ont été effectuées à l'aide d'un spectromètre d'absorption atomique Perkin Elmer 2380 équipé d'un four HGA 500 avec passeur automatique AS 40. Les méthodes d'analyses sont décrites dans Ouddane et al. (1992).
Les flux particulaires annuels entrant dans l'estuaire sont calculés à la station de May-sur-Orne à partir de la formule: $T=\Sigma M E S_{m j}{ }^{*} C_{x i}{ }^{*} V_{i}$ (Cossa et al., 1994) où $M E S_{m j}$, est la teneur en MES moyenne journalière calculée à partir de la relation $M E S_{i}=20,7^{\star} \operatorname{Ln}\left(Q_{i}\right)-40,6$ établie par modélisation statistique $\left(R^{2}=0,77\right)$ à partir des mesures instantanées (MES $S_{i}$ et $Q_{i}$ ) des MES et du débit; $C_{x i}$, teneur de l'élément $x$ dans les MES de l'échantillon instantané; $V_{i}$ : volume d'eau écoulé sur l'intervalle de temps pris autour de la date de mesure symétriquement entre deux prélèvements successifs.

\section{RÉSULTATS ET DISCUSSION}

\subsection{Evolution et caractérisation de la charge en suspension}

La charge moyenne en suspension à la sortie du bassin versant est relativement faible. Elle s'élève au cours des deux cycles humides étudiés à $42,1 \mathrm{mg} / \mathrm{l}$ en $1993-1994$ et à $62,9 \mathrm{mg} / \mathrm{l}$ en 1994-1995. La charge en suspension mesurée dans l'ensemble du bassin de l'Orne peut varier d'un facteur supérieur à 100 au cours de l'année, passant de moins de $1 \mathrm{mg} / \mathrm{l}$ en période d'étiage à plus de $120 \mathrm{mg} / \mathrm{l}$ en forte crue (tab. I).

L'évolution des teneurs en MES mesurées à l'exutoire du bassin (station N11 de May/Orne) suit globalement celle des débits liquides (fig. 1 et 2). L'exportation des sédiments se 
Tableau I. - Concentrations moyennes et extrèmes des matières particulaires en mg/l observées dans le bassin de l'Orne au cours des différentes périodes.

Table I. - Mean and extreme particulate matter concentrations $(\mathrm{mg} / \mathrm{l})$ in Orne basin.

Cycle période moyenne : minimum I maximum ।

E.T. $\quad \mathrm{CV}(\%)$ nombre d'échantillons

\begin{tabular}{|c|c|c|c|c|c|c|c|c|}
\hline \multirow{5}{*}{$\begin{array}{l}\text { exutoire } \\
\text { du bassin }\end{array}$} & 1993-94 & H.E. & 47.6 & & & & & \\
\hline & & cycle & 42.1 & 4 & 104 & 24.6 & 58.5 & 46 \\
\hline & & B.E. & 11.1 & & & & & \\
\hline & 1994-95 & H.E. & 64.6 & & & & & \\
\hline & & cycle & 62.9 & 3.5 & 125 & 29.6 & 47 & 47 \\
\hline $\begin{array}{l}\text { ensemble } \\
\text { (1994 et } 1\end{array}$ & $\begin{array}{l}\text { Uu bassin } \\
95 \text { ) }\end{array}$ & & 22,6 & 0.2 & 122.2 & 24 & 106 & 253 \\
\hline
\end{tabular}

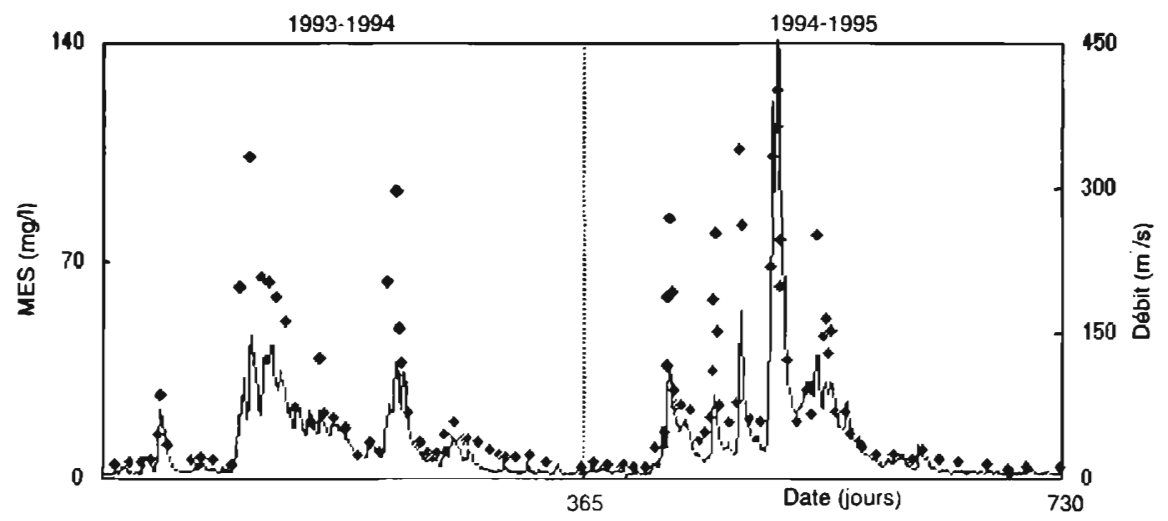

Fig. 1. - Evolution des teneurs en MES mesurées dans l'Orne (cycles hydrologiques 1993-1995) Fig. 1. - Evolution of the suspended matters concentrations (SPM) in the Orne (1993-1995)

fait principalement durant les périodes de crue qui correspondent en général aux phases de ruissellement superficiel sur les versants. Les premières crues de l'année sont très érosives, les augmentations importantes des teneurs en MES étant dues à la remise en mouvement de tous les matériaux meubles des versants et surtout de ceux déposés sur le fond de la rivière.

Les variations spatio-temporelles des teneurs en MES mettent en évidence la différence de comportement des particules entre les périodes d'étiage et de crue sur l'Orne et ses affluents (fig. 3 ) : 

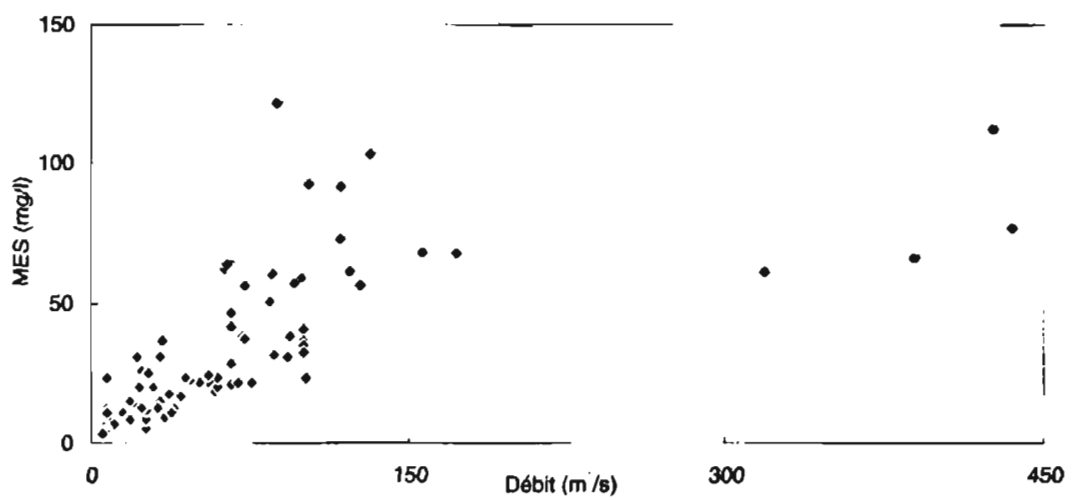

Fig. 2. - Variations des MES avec le débit dans l'Orne (1993-1995)

Fig. 2. - SPM-water discharge relationship in the Orne (1993-1995)

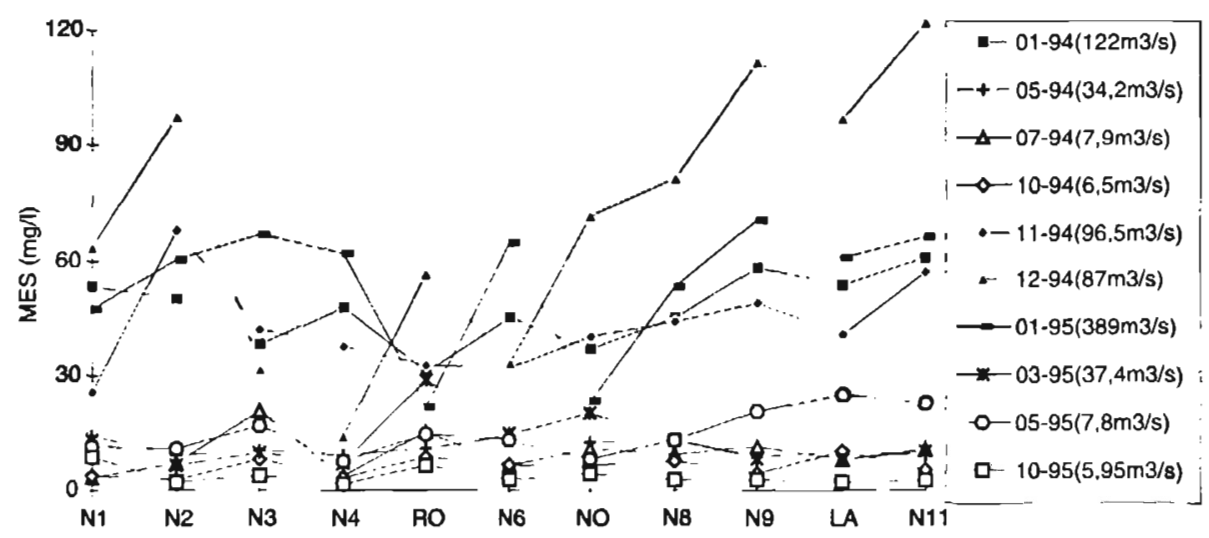

Fig. 3. - Variations amont-aval des concentrations de MES dans le bassin versant de l'Orne Fig. 3. - Upstream-downstream variations of the SPM concentrations in the Orne basin

- la période de basses eaux est caractérisée par des faibles écarts de matière solide transportée en poids et en turbidité. Le faible débit et l'absence de précipitation limitent les écarts de matière transportée sur l'ensemble du fleuve. Le développement planctonique est la source principale de particules en suspension, ce qui implique que la concentration varie essentiellement en fonction de la température et de la présence d'éléments nutritifs bien plus qu'en fonction de l'environnement lithologique et hydrodynamique. La charge en MES varie entre 0,2 et $26,5 \mathrm{mg} / \mathrm{l}$ sur l'ensemble des stations de prélèvement. Les échantillons correspondant 
à cette période montrent au MEB une flore très riche en espèces planctoniques oligahalobes qui peuvent représenter jusqu'à $95 \%$ du total. Parmi ces espèces Cyclostéphanos dubius (espèce planctonique commune dans les eaux plus ou moins polluées) prédomine en été et automne dans tous les échantillons. Asterionella formosa, espèce planctonique relativement sensible à la pollution, montre une présence discontinue en été. Les spectres granulométriques correspondant à cette période mettent notamment en évidence le rôle joué par le barrage de Rabodanges dans le transport et la nature des particules compte tenu de l'intense activté phytoplanctonique et des phénomènes de décantation qui s'y produisent (fig. 4.a). Ainsi, par exemple, la prédominance dans le lac des classes 9 et $12 \mu \mathrm{m}$, correspondant à deux générations de Cyclostéphanos dubius, va se retrouver jusqu'à l'exutoire.

- la période des hautes eaux est caractérisée par une forte variabilité d'amont en aval de la turbidité et des MES et notamment lors des crues qui sont généralement provoquées par des épisodes pluvieux intenses de localisation restreinte. La charge solide peut alors atteindre des valeurs supérieures à $100 \mathrm{mg} / \mathrm{l}$. Les terrains calcaires mieux lessivés, fournissent généralement les concentrations en MES les plus importantes. Une baisse des concentrations est observée lors de la crue de janvier 1995 par rapport à décembre 1994 due probablement au phénomène de dilution.
Les spectres granulométriques montrent l'évolution importante de la distribution des populations en répartition et en concentration au cours de cette période (fig. 4.b et c). En effet, on observe un étalement général des spectres, les tailles des groupes de population passant de $3-16 \mu \mathrm{m}$ en basses eaux à 3-35 $\mu \mathrm{m}$ en hautes eaux. Cet étalement vers les grandes tailles correspond probablement à l'arrivée de petits agrégats de sols associés aux processus d'érosion des sols. Les affluent Rouvre et Noireau dégagent lors des hautes eaux d'importantes masses de particules, mais en fin de crue (01/95), ils se comportent comme des réducteurs d'apports particulaires et entraînent une dilution des concentrations en MES. On constate par ailleurs une diminution des tailles des groupes de population en fin de crue due soit à une décantation préalable plus importante, soit à une réduction des tailles à la source, lors de l'arrachement, les particules les plus grossières qui offrent le plus de prise à l'érosion ayant déjà été déplacées lors de la crue précédente.

\subsection{Composition chimique des MES}

La composition chimique des sédiments transportés par les fleuves, reflète généralement la composition chimique des sols et des roches du bassin versant. Dans les tableaux II et III, sont reportées à titre de comparaison, la composition chimique 



Fig. 4. - Spectres granulométriques de divers campagnes de basses et hautes eaux

Fig. 4. - Some sampling granulometric spectra 
Tableau II. - Comparaison des compositions chimiques moyennes des matériaux en suspension de l'Orne à divers fleuves français et à la moyenne mondiale

Table II. - Comparison of the major metals concentrations (\%) in suspended sediment between Orne, some french rivers and world average

références: 1 : Martin et Withfield (1983); 2 : Martin et Meybeck (1979); 3 : Snoussi (1988); 4 : Etchanchu (1988); 5 : cette étude (1993-1995).

\begin{tabular}{cccccccccc}
\multicolumn{2}{c}{$\begin{array}{c}\text { Elément moyenne } \\
\text { en } \% \text { Lo }\end{array}$} & Loire & Meuse & Rhin & Rhône & Adour & Garonne & Girou & Orne \\
$\mathrm{Si}$ & 18.2 & 38.3 & 38.6 & 36.4 & 29.6 & 42 & 36.8 & 32.4 & 33.3 \\
$\mathrm{AL}$ & 4.97 & 9.73 & 7.51 & 7.5 & 5.29 & 6.08 & 11.85 & 10 & 6.9 \\
$\mathrm{Fe}$ & 3.36 & - & - & - & - & 3.36 & 2.31 & 6.22 & 4.8 \\
$\mathrm{Ti}$ & 0.34 & 0.53 & 0.69 & 0.54 & 0.29 & 0.37 & 0.50 & 0.54 & 0.35 \\
$\mathrm{Ca}$ & 1.53 & 4.71 & 3.43 & 7.28 & 13.07 & 2.61 & 1.95 & 7.93 & 2.6 \\
$\mathrm{Mg}$ & 0.71 & 1.5 & 1.02 & 1.78 & 1.17 & 0.72 & 1.74 & 1.87 & 1.22 \\
$\mathrm{Na}$ & 0.35 & 0.51 & 0.43 & 0.06 & 0.5 & 0.29 & 0.37 & 0.44 & 0.68 \\
$\mathrm{~K}$ & 1.24 & 2.34 & 1.49 & 0.81 & 1.32 & 1.07 & 2.5 & 3.04 & 1.82 \\
& $(1)$ & & & $(2)$ & & $(3)$ & & $(4)$ & $(5)$
\end{tabular}

Tableau III. - Concentrations moyennes en $\mu \mathrm{g} / \mathrm{g}$ des éléments traces particulaires dans les eaux de l'Orne et dans certaines rivières françaises.

Table III. - Heavy metal concentrations $(\mu \mathrm{g} / \mathrm{g})$ in suspended sediments from Orne, some french rivers and world average.

\begin{tabular}{cccccccl} 
& $\mathrm{Cd}$ & $\mathrm{Cu}$ & $\mathrm{Cr}$ & $\mathrm{Pb}$ & $\mathrm{Zn}$ & $\mathrm{Ni}$ & \multicolumn{1}{c}{ références } \\
Orne & 2.8 & 80 & 65 & 72 & 235 & 52 & cette étude \\
Adour & - & 58 & - & 60 & 162 & - & Snoussi, 1988 \\
Girou & - & 33 & 75 & - & 160 & 40 & Etchanchu, 1988 \\
Garonne & - & 51 & 255 & - & 874 & 33 & Meybeck, 1984 \\
Seine & 4.95 & 174 & - & 184 & 611 & - & Idlafkih et al., 1995 \\
Rhin & 32 & 260 & - & 425 & 1600 & - & V.Weijden et al., 1989 \\
Huanghe & 0.18 & 26.7 & 76.9 & 16.4 & 69.8 & 40.3 & Zhang et al., 1995 \\
Léna & - & 28 & - & 23 & 143 & - & in Cossa et al., 1994 \\
moyenne mondiale & 1 & 100 & 100 & 100 & 250 & 90 & Martin et Whitfield, 1983
\end{tabular}

moyenne mesurée des MES transportés par l'Orne au cours des deux cycles étudiés, celles de quelques fleuves français et les moyennes mondiales.
Les teneurs en métaux majeurs mesurées des suspensions de l'Orne sont globalement comparables à celle des sédiments transportés par les fleuves français, mais 
différent notablement, pour certains éléments du moins, de celle de la moyenne mondiale donnée par Martin et Withfield (1983). Le rapport $\mathrm{R}$ $\left(\mathrm{R}=\mathrm{SiO}_{2} / \mathrm{Al}_{2} \mathrm{O}_{3}+\mathrm{Fe}_{2} \mathrm{O}_{3}\right)$ est égal à 2,62 pour l'Orne. Cette valeur se situe dans la plage des valeurs calculées pour certains fleuves français: Moselle et le Girou $(2,25)$; Rhône $(2,95)$; Rhin $(2,38)$; Loire $(2,29)$; Adour $(2,85)$ et Garonne $(2,36)$.

Comparées aux moyennes des teneurs dans des fleuves considérés peu affectés par les apports anthropiques (Huanghe et Léna), les teneurs en métaux traces des MES présentent de fortes concentrations en $\mathrm{Cd}, \mathrm{Cu}, \mathrm{Zn}$ et $\mathrm{Pb}$. Le $\mathrm{Cr}$ et le $\mathrm{Ni}$ présentent des teneurs comparables. Néanmoins, sauf pour le $\mathrm{Cd}$, ces valeurs restent plus faibles que la composition moyenne des rivières donnée par Martin et Whitfield (1983). Cette pollution des eaux en Cd est confirmée par le facteur d'enrichissement (FE) calculé à partir du rapport des teneurs dans les sédiments de fond et dans les substrats normalisées à Al $\left(\mathrm{C}_{\text {MES }} / \mathrm{C}_{\text {MES-Al }}\right) /\left(\mathrm{C}_{\text {SUB }} / \mathrm{C}_{\text {SUB-Al }}\right)$. Nous avons utilisé les teneurs dans les sédiments au lieu de celles dans les MES, car les sédiments peuvent fournir outre un aperçu de l'historique de la pollution dans la riviere, des informations importantes sur le comportement de la rivière dans son ensemble (Windom et al., 1989; Zhang et al., 1995). II faut cependant noter que le transport solide dans les rivières s'effectue essentiellement en suspension et que la minéralogie/taille des particules et/ou la composition chimique de ces suspensions peut différer notablement de celles des sédiments de fond. La plupart des valeurs obtenues sont comprises entre 0,8 et 1,2 ce qui indique un enrichissement faible à nul (tab. IV). Ce comportement caractérise des apports en provenance de l'érosion du bassin de drainage. Certains métaux montrent des valeurs de FE plus éparses dues probablement à la grande diversité des matériaux de références utilisés dans les cal-

Tableau IV. - Facteur d'enrichissement (FE) des métaux traces dans les sédiments de fond en différentes stations de l'Orne

Table IV. - Enrichement factors (EF) of heavy metals in bottom sediments from some Orne gauging stations

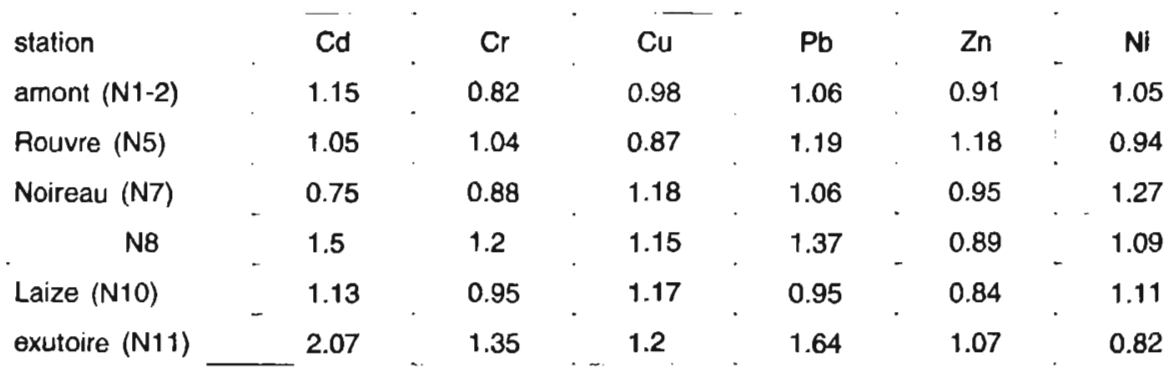


Tableau V. - Evolution amont-aval des concentrations moyennes des éléments majeurs et traces particulaires dans le bassin versant de l'Orne (1994-1995).

Table V. - Upstream-downstream evolution of the mean concentrations of the major and trace particulate elements in the Orne basin (1993-1995)

\begin{tabular}{|c|c|c|c|c|c|c|c|c|c|c|c|c|c|c|}
\hline \multirow[b]{2}{*}{ Stations } & \multicolumn{8}{|c|}{ Métaux majeurs (\%) } & \multicolumn{4}{|c|}{ métaux traces $(\mu \mathrm{g} / \mathrm{g})$} & \multicolumn{2}{|c|}{ 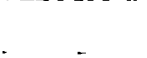 } \\
\hline & $\mathrm{Si}$ & Al & $\mathrm{Fe}$ & $\mathrm{Ca}$ & $\mathrm{Mg}$ & $\mathrm{Na}$ & K & $\mathrm{Ti}$ & $\mathrm{Cd}$ & $\mathrm{Cu}$ & $\mathrm{Cr}$ & $\mathrm{Pb}$ & $\mathrm{Zn}$ & $\mathbf{N i}$ \\
\hline $\begin{array}{l}\text { N1-2 } \\
\text { N5 } \\
\text { N7 } \\
\text { N10 } \\
\text { N11 }\end{array}$ & $\begin{array}{l}33.8 \\
37.5 \\
36.1 \\
32.6 \\
32.4\end{array}$ & $\begin{array}{l}5.3 \\
8.1 \\
7.8 \\
5.3 \\
6.7\end{array}$ & $\begin{array}{l}4.5 \\
5.5 \\
4.0 \\
3.9 \\
4.6\end{array}$ & $\begin{array}{l}1.39 \\
0.48 \\
0.50 \\
1.27 \\
1.19\end{array}$ & $\begin{array}{l}0.88 \\
0.99 \\
1.05 \\
0.83 \\
1.01\end{array}$ & $\begin{array}{l}0.32 \\
0.55 \\
0.60 \\
0.39 \\
0.50\end{array}$ & $\begin{array}{l}1.81 \\
1.72 \\
1.76 \\
1.79 \\
2.07\end{array}$ & $\begin{array}{l}0.22 \\
0.41 \\
0.31 \\
0.25 \\
0.30\end{array}$ & $\begin{array}{l}1.71 \\
1.69 \\
1.64 \\
1.80 \\
2.60\end{array}$ & $\begin{array}{l}75 \\
67 \\
80 \\
70 \\
76\end{array}$ & $\begin{array}{l}62.5 \\
56.1 \\
64.2 \\
59.3 \\
63.8\end{array}$ & $\begin{array}{l}53.4 \\
48.9 \\
57.4 \\
51.8 \\
58.5\end{array}$ & $\begin{array}{l}187 \\
168 \\
198 \\
179 \\
195\end{array}$ & $\begin{array}{l}51.3 \\
62.5 \\
52.2 \\
49.1 \\
54.3\end{array}$ \\
\hline $\begin{array}{l}\text { moyenne } \\
\text { minimum } \\
\text { maximum } \\
\text { Ecart type } \\
\text { CV (\%) }\end{array}$ & $\begin{array}{l}33.7 \\
32.2 \\
37.4 \\
1.65 \\
4.9\end{array}$ & $\begin{array}{l}6.1 \\
4.8 \\
8.1 \\
0.39 \\
6.4\end{array}$ & $\begin{array}{l}4.4 \\
3.8 \\
5.5 \\
0.33 \\
7.5\end{array}$ & $\begin{array}{l}1.2 \\
0.4 \\
1.8 \\
0.1 \\
8.5\end{array}$ & $\begin{array}{c}0.9 \\
0.8 \\
1.1 \\
0.07 \\
7\end{array}$ & $\begin{array}{l}0.43 \\
0.3 \\
0.6 \\
0.04 \\
8.6\end{array}$ & $\begin{array}{l}1.81 \\
1.63 \\
2.13 \\
0.15 \\
8.1\end{array}$ & $\begin{array}{l}0.27 \\
0.20 \\
0.41 \\
0.02 \\
7.2\end{array}$ & $\begin{array}{c}1.87 \\
1.56 \\
2.60 \\
0.22 \\
12\end{array}$ & $\begin{array}{l}74 \\
67 \\
80 \\
3.2 \\
4.4\end{array}$ & $\begin{array}{r}61.0 \\
56.2 \\
64.2 \\
3.1 \\
5.0\end{array}$ & $\begin{array}{c}54 \\
45 \\
60 \\
4.9 \\
9.2\end{array}$ & $\begin{array}{l}185 \\
168 \\
198 \\
9.4 \\
5.1\end{array}$ & \begin{tabular}{r}
\multicolumn{1}{r}{54} \\
48.6 \\
62.5 \\
4.6 \\
8.5
\end{tabular} \\
\hline
\end{tabular}

culs. On trouve pour le $\mathrm{Cd}$ une valeur de 2,07 à l'exutoire confirmant la pollution des eaux par ce métal.

Les éléments les plus abondants dans les MES de l'Orne à son exutoire sont par ordre décroissant :

$$
\begin{aligned}
\mathrm{Si} & >\mathrm{Al}>\mathrm{Fe}>\mathrm{Ca}>\mathrm{K}>\mathrm{Mg}>\mathrm{Na} \mathrm{Ti} \\
\text { et } \mathrm{Zn} & >\mathrm{Cu}>\mathrm{Pb}>\mathrm{Cr}>\mathrm{Ni}>\mathrm{Cd}
\end{aligned}
$$

$\mathrm{Si}, \mathrm{Al}$ et $\mathrm{Fe}$ représentent à eux trois $87 \%$ de la somme de ces éléments.

Le tableau $\mathrm{V}$ donne pour les quatre stations représentatives des principaux sous-bassins (amont: N1-2, Rouvre: N5, Noireau: N7 et Laize: $\mathrm{N} 10$ ) et pour la station à l'exutoire $\mathrm{N} 11$, les statistiques générales sur les concentrations mesurées des éléments étudiés.

Les variations amont-aval des concentrations des différents éléments majeurs sont globalement conformes à la répartition zonale de ces éléments dans les substrats composant le bassin. Elles mettent notamment en évidence la provenance de $\mathrm{Si}, \mathrm{Al}$ et $\mathrm{Na}$ des bassins silico-alumineux de la Rouvre et du Noireau, et la provenance de $\mathrm{Ca}$ des bassins calcaires de l'amont et de la Laize. Pour les métaux en traces, $\mathrm{Cr}$ et $\mathrm{Zn}$ proviennent essentiellement de l'amont et du Noireau, $\mathrm{Cu}$ du Noireau et Ni de la Rouvre. Cette évolution amont-aval de la composition chimique moyenne sur le cours principal, ne montre pas de tendance particulière (faibles valeurs $d u$ coefficient de variation), si ce n'est les légères augmentations observées pour $\mathrm{Pb}$ et $\mathrm{Zn}$. Ceci peut être significatif d'une relative homogénéité de la charge solide transportée par les eaux: les variations des concentrations des métaux particulaires dans l'Orne dépassent rarement un facteur de 1,5. L'augmentation des concentrations en plomb vers l'aval peut être due à l'augmentation de la densité du réseau routier. 

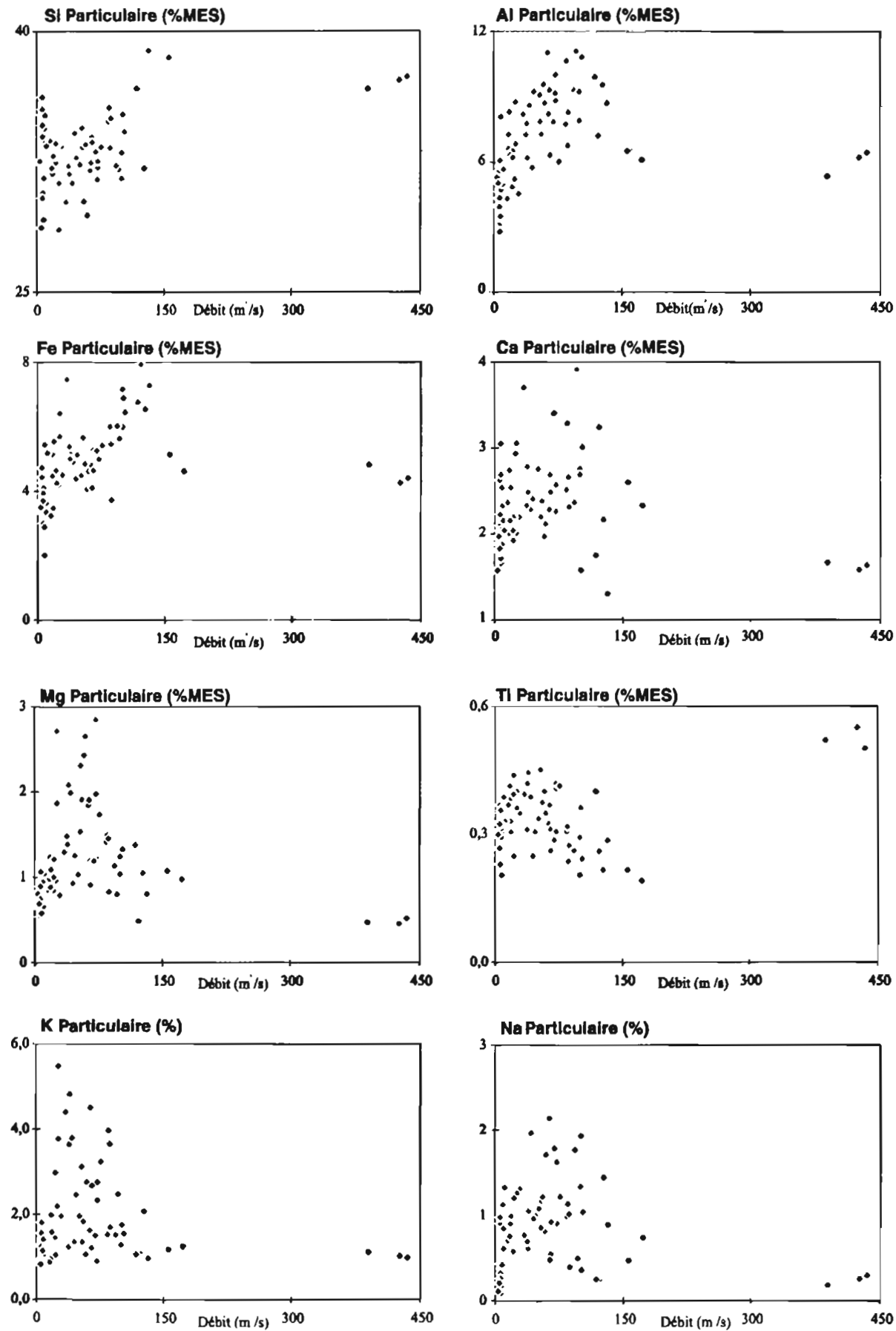

Fig. 5. - Variations des teneurs en éléments majeurs dans les suspensions avec le débit à May/Orne Fig. 5. - Variations of the major elements contents in Orne SPM (1993-1995) 
A. Nasseh, H. Texier, M. Lacroix et B. Ouddane
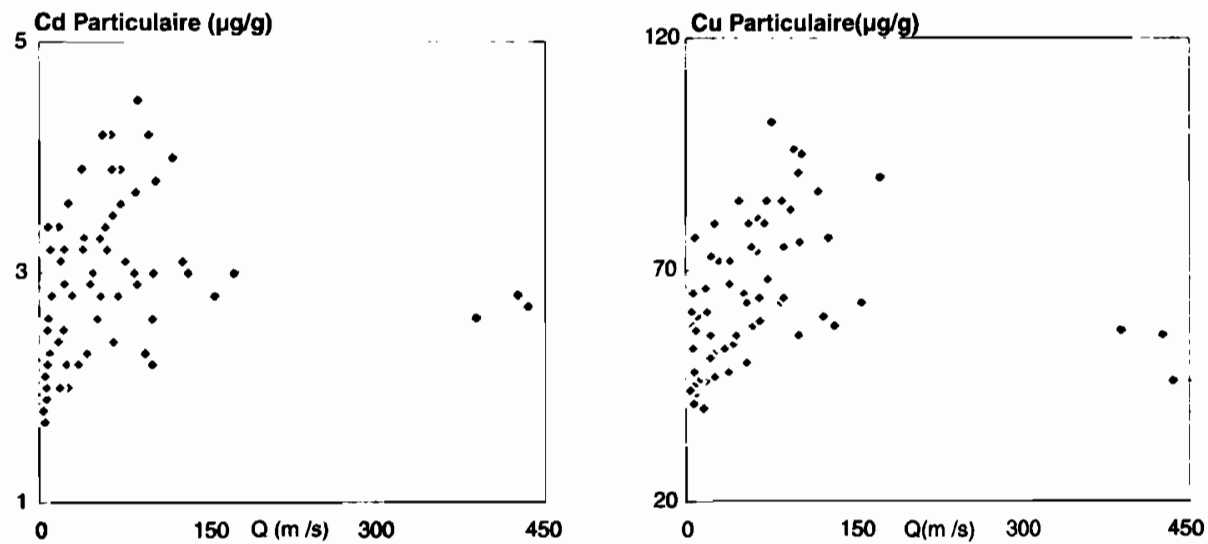

120 Cr Particulaire $(\mu g / g)$
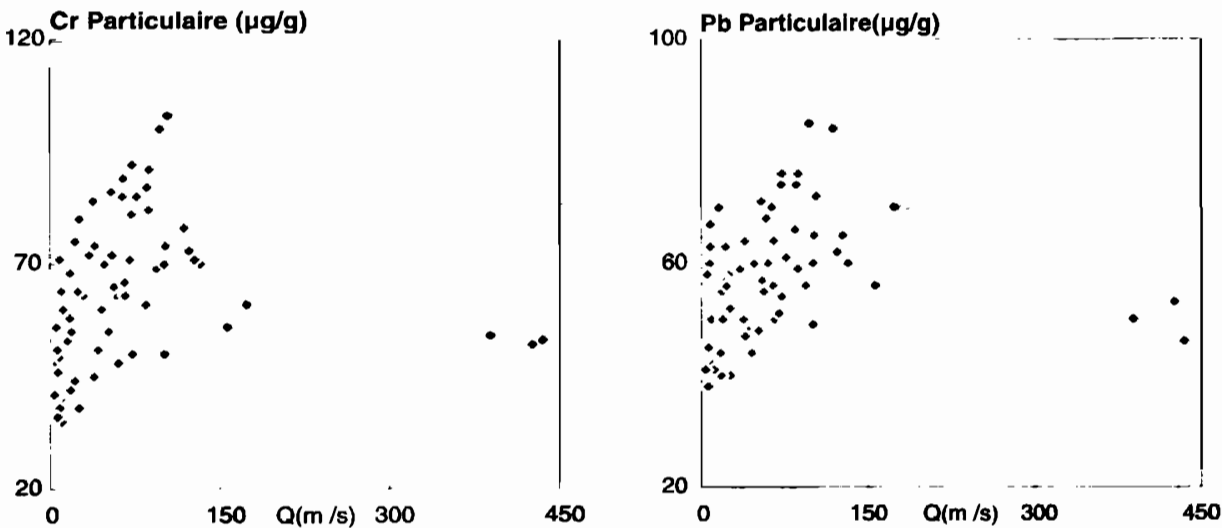

310 Zn Particulaire $(\boldsymbol{\mu g} / \mathbf{g})$
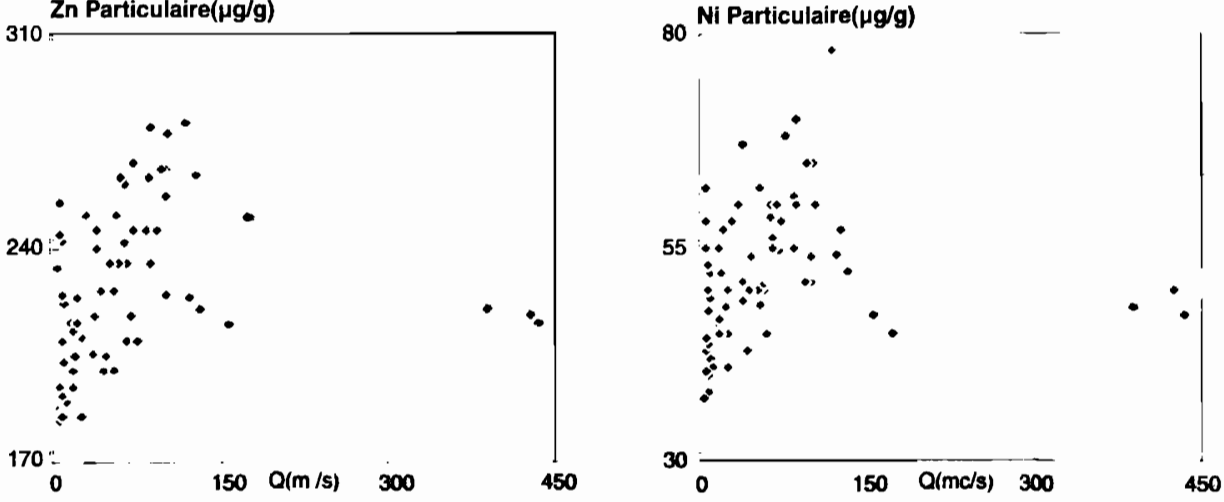

Fig. 6. - Variations des teneurs en métaux en traces des MES avec le débit à May/Orne (1993-95) Fig. 6. - Variations of the trace metals contents in Orne SPM (1993-1995) 
Mis à part le calcium, tous les autres éléments voient leurs concentrations diminuer significativement en étiage dans le barrage de Rabodanges où la décantation des particules en suspension est très importante. $\mathrm{Ca}$ présente en cette période des teneurs importantes à cette station dues à la précipitation de la calcite compte tenu des conditions physico-chimiques favorables à cette précipitation $(\mathrm{pH}>9$; $t^{\circ} \geq 25^{\circ} \mathrm{C}$ ). Les variations saisonnières des teneurs dans les MES dépendent des variations du débit et des variations de la charge solide. Globalement pour les éléments dont le transport est généralement associé aux MES ( $\mathrm{Al}, \mathrm{Fe}$ et métaux traces), les concentrations particulaires augmentent avec le débit jusqu'à un seuil de $110-120 \mathrm{~m}^{3} / \mathrm{s}$ (fig. 5 et 6 ). Les variations observées pour les autres éléments $(\mathrm{Ca}, \mathrm{Mg}, \mathrm{Na}, \mathrm{K})$, transportés généralement sous forme dissoute, montrent un nuage de points plus ou moins dispersé. Ces augmentations des teneurs avec le débit montrent la prédominance des processus d'apports métalliques détritiques du bassin versant. Une diminution générale des concentrations lorsque les débits dépassent le seuil de $110-120 \mathrm{~m}^{3} / \mathrm{s}$ est observée pour l'ensemble des métaux, sauf pour $\mathrm{Si}$ et $\mathrm{Ti}$. Cette diminution des concentrations avec le débit, observée dans de nombreux bassins, est souvent attribuée à des processus de dilution conséquence de l'augmentation du débit liquide, du mélange des sédiments pauvres en métaux érodés du bassin versant ou de l'effet des sédiments en suspension plus grossiers qui sont plus abondants en période de crue. Les concentrations les plus importantes des éléments correspondent généralement aux situations hydrologiques de début de crue, montrant que le transport de ces éléments est très important pendant cette période. Des concentrations relativement élevées sont mesurées pendant certaines campagnes estivales. Elles peuvent être en relation avec des processus chimiques d'enrichissement des MES, comme conséquence de l'adsorption et/ou de la complexation plus intense des métaux favorisée par un temps de résidence plus long en condition de faible débit.

La transcription des relations débitconcentration en termes de flux instantanés en fonction des débits montre que les flux instantanés des métaux particulaires, augmentent nettement avec le débit et que les apports à l'exutoire du bassin s'effectuent essentiellement lors des crues. Ces flux ne sont que faiblement affectés par la dilution des concentrations lors des crues et par la diminution des teneurs dans les suspensions (fig. 7 et 8 ). Ceci est due essentiellement aux importants ordres de grandeur dans lesquels varient les débits et les concentrations des MES par rapport à ceux beaucoup plus faibles des teneurs dans les MES.

\subsection{Traitement statistique des données}

Une analyse en composantes principales a été menée en utilisant en plus des concentrations des métaux 

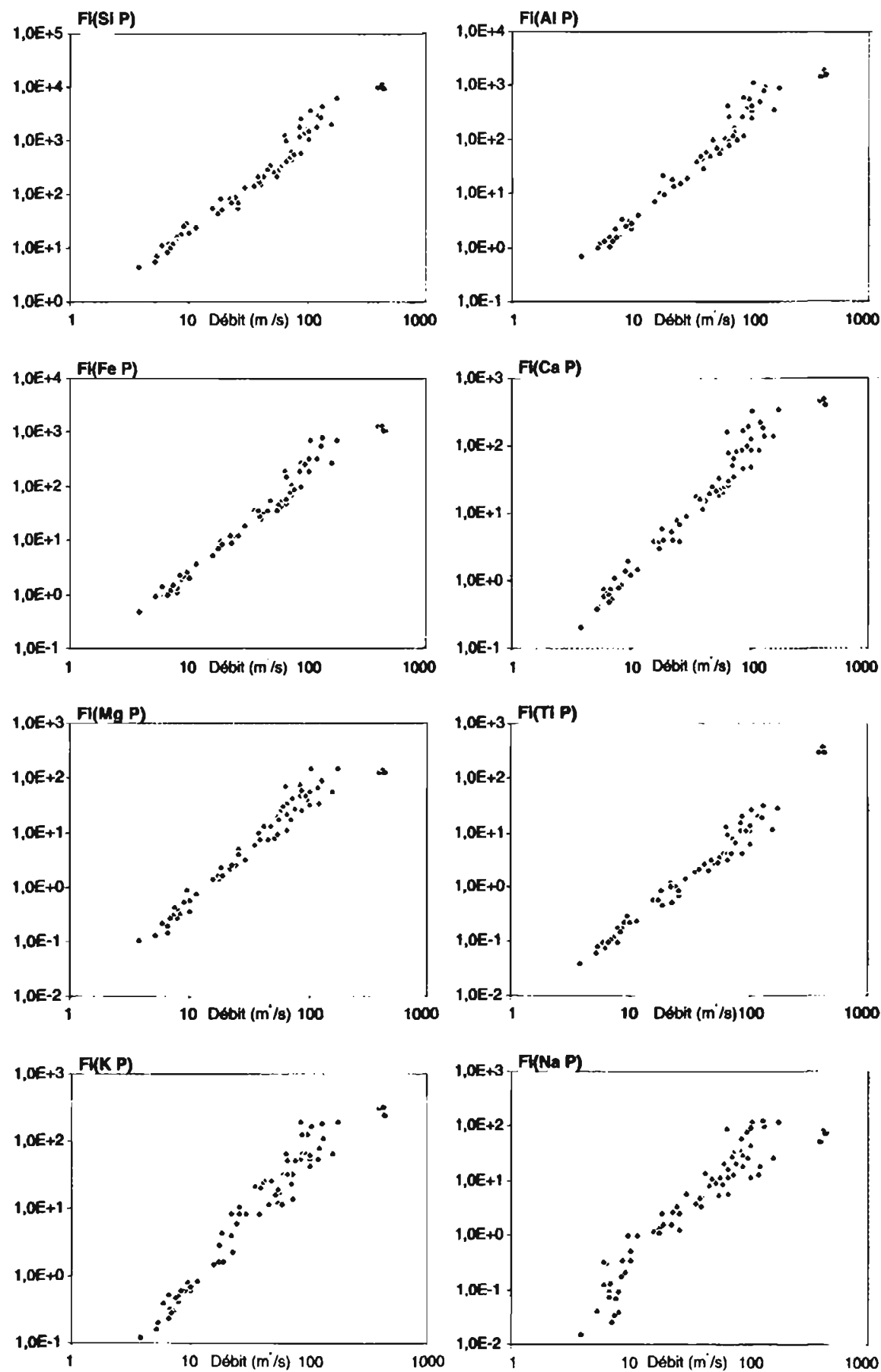

Fig. 7. - Variations des flux instantanés des métaux majeurs particulaires $(\mathrm{g} / \mathrm{s})$ avec le débit à May/Orne.

Fig. 7. - Instantaneous particulate major element flux Fi vs river discharge 

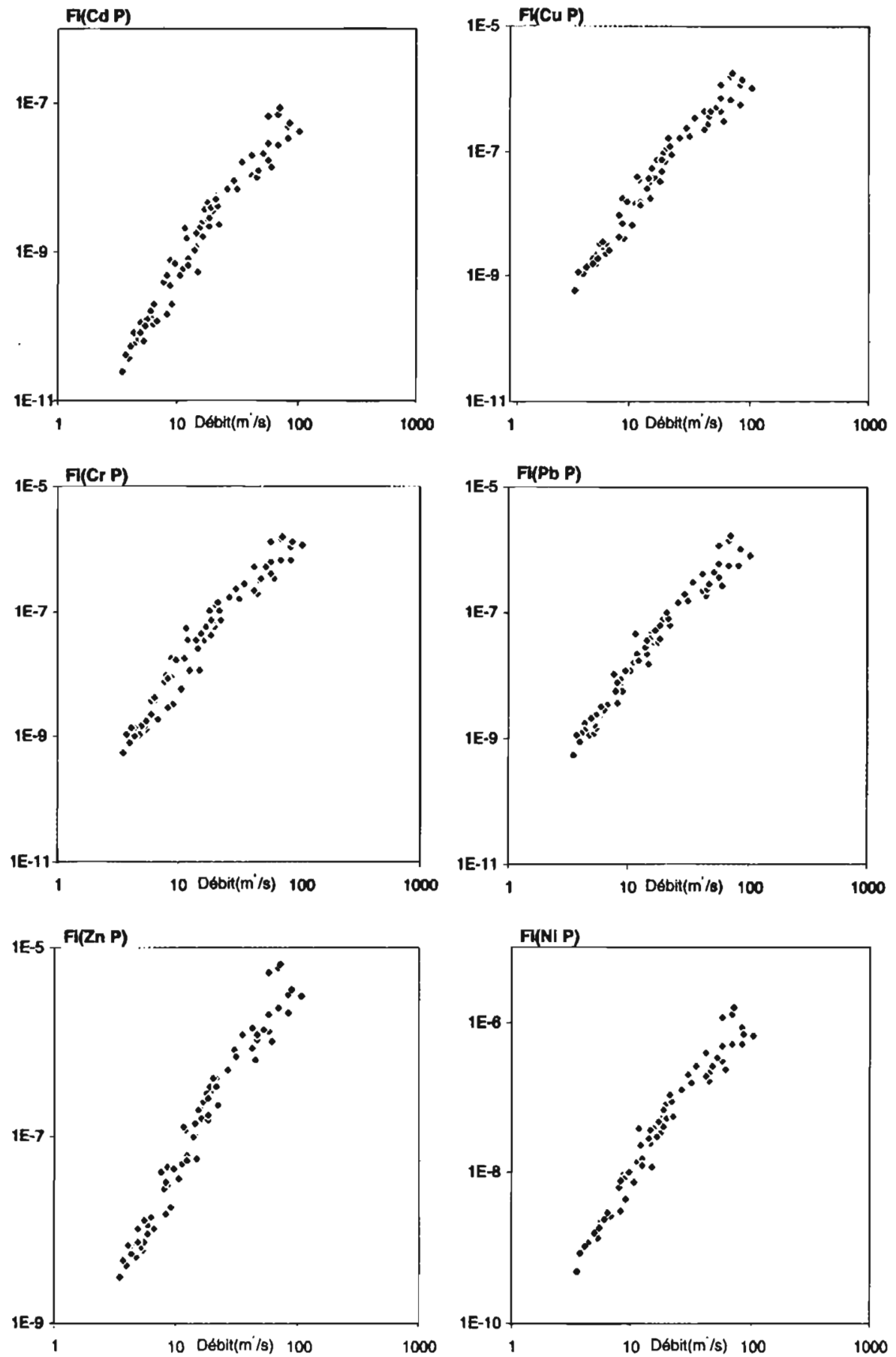

Fig. 8. - Variations des flux instantanés (Fi) des métaux en traces particulaires dans les eaux de l'Orne (1993-1995) (coordonnées semi-logarithmiques)

Fig. 8. - Instantaneous particulate trace metal flux vs river discharge 
Tableau VI. - Analyse en composantes principales (ACP) matrice de corrélation Table VI. - Correlation matrix of the principal component analysis (ACP)

\begin{tabular}{|c|c|c|c|c|c|c|c|c|c|c|c|c|c|c|c|c|}
\hline & $Q$ & MES & COP & Si & Al & $\mathrm{Fe}$ & $\mathrm{Ca}$ & $\mathbf{M g}$ & $K$ & $\mathrm{Na}$ & $\mathrm{Ti}$ & Cd & $\mathrm{Cr}$ & $\mathrm{Cu}$ & $\mathrm{Pb}$ & $\mathrm{Zn}$ \\
\hline MES & 0.84 & • & & & & & & & & & & & & & & \\
\hline COP & 0.81 & 0.80 & * & & & & & & & & & & & & & \\
\hline $\mathrm{Si}$ & 0.50 & 0.42 & 0.17 & - & & & & & & & & & & & & \\
\hline Al & 0.86 & 0.84 & 0.28 & 0.13 & • & & & & & & & & & & & \\
\hline $\mathrm{Fe}$ & 0.70 & 0.72 & 0.39 & 0.06 & 0.88 & • & & & & & & & & & & \\
\hline $\mathrm{Ca}$ & 0.31 & 0.28 & 0.31 & -0.23 & 0.01 & 0.10 & $*$ & & & & & & & & & \\
\hline $\mathrm{Mg}$ & 0.50 & 0.43 & 0.28 & 0.15 & 0.43 & 0.46 & 0.15 & - & & & & & & & & \\
\hline $\mathrm{K}$ & 0.33 & 0.28 & 0.43 & 0.05 & 0.35 & 0.22 & 0.56 & 0.20 & " & & & & & & & \\
\hline $\mathrm{Na}$ & 0.42 & 0.35 & 0.18 & 0.38 & 0.46 & 0.40 & 0.10 & 0.40 & 0.38 & $*$ & & & & & & \\
\hline $\mathrm{Ti}$ & 0.44 & 0.48 & 0.22 & 0.57 & 0.33 & 0.31 & 0.04 & 0.23 & -0.10 & 0.33 & • & & & & & \\
\hline $\mathrm{Cd}$ & 0.74 & 0.70 & 0.32 & 0.18 & 0.26 & 0.19 & 0.52 & 0.13 & 0.22 & 0.13 & 0.08 & • & & & & \\
\hline $\mathrm{Cr}$ & 0.75 & 0.81 & 0.62 & 0.10 & 0.71 & 0.63 & 0.16 & 0.15 & 0.06 & 0.10 & 0.12 & 0.08 & - & & & \\
\hline $\mathrm{Cu}$ & 0.80 & 0.78 & 0.59 & 0.18 & 0.68 & 0.67 & 0.07 & 0.23 & 0.16 & 0.19 & 0.17 & 0.13 & 0.60 & • & & \\
\hline $\mathrm{Pb}$ & 0.48 & 0.51 & 0.29 & 0.09 & 0.18 & 0.17 & 0.20 & 0.15 & 0.03 & 0.23 & 0.05 & 0.26 & 0.24 & 0.09 & - & \\
\hline $\mathrm{Zn}$ & 0.49 & 0.76 & 0.52 & 0.20 & 0.61 & 0.75 & 0.04 & 0.20 & 0.21 & 0.21 & 0.13 & 0.12 & 0.69 & 0.62 & 0.10 & - \\
\hline $\mathrm{Ni}$ & 0.52 & 0.58 & 0.18 & 0.27 & 0.26 & 0.12 & 0.18 & 0.08 & 0.06 & 0.07 & 0.24 & 0.30 & 0.15 & 0.13 & 0.27 & 0.15 \\
\hline
\end{tabular}

particulaires, le débit, la MES et le carbone organique particulaire (COP). La matrice et les cercles de corrélation sont donnés dans le tableau $\mathrm{VI}$ et la figure 9. Les matrices de corrélation montrent que la MES présente une bonne corrélation avec la plupart des métaux dont le transport s'effectue principalement sous forme particulaire. $\mathrm{Ti}$ qui présente sa meilleure corrélation avec $\mathrm{Si}$, semble être lié dans une large mesure à l'exportation du quartz et notamment dans les fractions granulométriques les plus grossières. $\mathrm{Fe}, \mathrm{Cr}, \mathrm{Cu}$ et $\mathrm{Zn}$ associés à Al sont donc liés en grande partie à la fraction argileuse. Les minéraux argileux en suspension servent dans les eaux de surface à la fois de moyen de transport et de réservoir des métaux lourds. $\mathrm{Cr}$ et $\mathrm{Cu}$ montrent par ailleurs de légères corrélations avec le COP. Ces deux métaux apparaissent en conséquence complexés en partie avec les molécules organiques. La matière organique dans les milieux fluviatiles, joue un rôle important dans le piégeage et la fixation des éléments traces suivant des processus très complexes et qui dépendent des conditions physicochimiques et des supports géochimiques des matériaux en suspension (Weiss et al., 1991; Baisch, 1994). Zn présente sa meilleurs corrélation avec Fe. Les oxy-hydroxydes de fer sont souvent considérés comme les substrats les plus importants pour l'ad- 


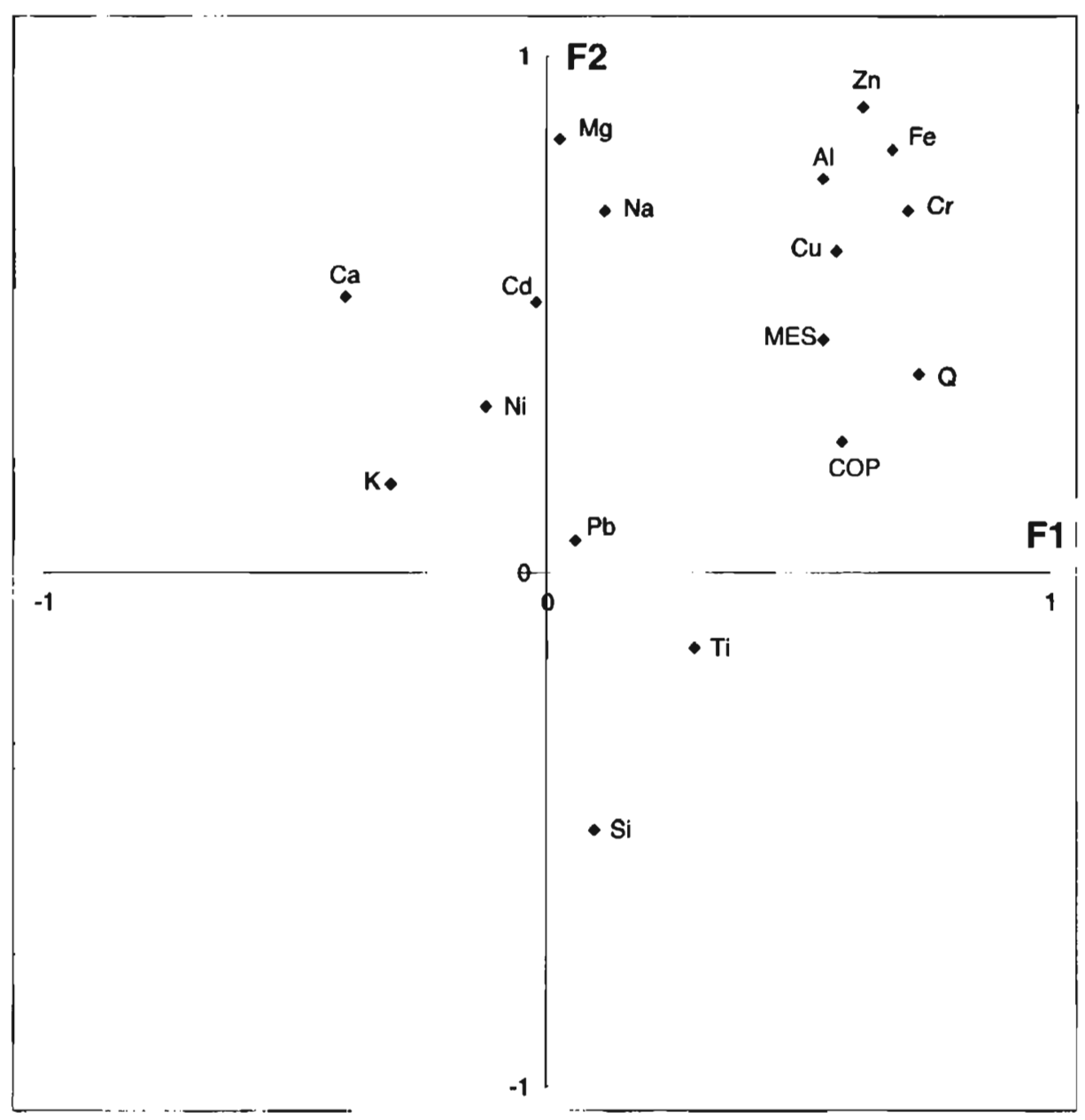

Fig. 9. - Cercles de corrélation de l'ACP plan F1, F2 sur 17 variables

Fig. 9. - Correlation circle: plan F1, F2 for 17 variables

sorption de Zn (Johnson, 1986; Nirel, 1987; Baisch, 1994). Cd présente la meilleure corrélation avec $\mathrm{Ca}$.

Dans les cercles de corrélation, seuls les axes $\mathrm{F} 1$ et $\mathrm{F} 2$ ont été représentés (fig. 9). Leurs contributions respectives à l'inertie totale du nuage sont de $51 \%$ pour $\mathrm{F} 1$ et $14,8 \%$ pour
F2. Ces cercles mettent d'abord en évidence la bonne corrélation existant entre les métaux particulaires et la MES. L'axe F1 montre d'une part l'opposition entre Si et la plupart des éléments traces, et d'autre part les corrélations positives entre $\mathrm{Al}, \mathrm{Fe}$, la plupart des éléments traces et le 
COP. Cet axe montre donc que la fraction argileuse fine est riche en métaux traces et suit l'évolution des teneurs en matières organiques, tandis que les fractions grossières associées au quartz sont elles pauvres en éléments traces. L'axe F2 met en évidence l'origine des différents éléments, avec d'une part les éléments fournis essentiellement par les terrains calcaires ( $\mathrm{Ca}$ notamment), et d'autre part les éléments fournis par les terrains schisteux ( $\mathrm{Si}, \mathrm{Al}, \mathrm{Na}$ et Mg notamment).

\subsection{Bilans des transports}

Les estimations calculées dans cette étude constituent les premières estimations moyennes des apports solides dans le bassin de l'Orne, aucune étude générale relative à l'érosion et aux transports solides dans le bassin de l'Orne n'ayant été effectuée auparavant. Les flux annuels des différents éléments apportés par l'Orne à l'entrée de son système estuarien ainsi que les flux spécifiques calculés pour les deux cycles hydrologiques sont donnés dans le tableau VII. Les flux exportés sont plus importants lors du cycle 1994-1995 plus humide. Le bilan annuel des transports de matières en suspension, par l'Orne à May/Orne est estimé à plus de $63.10^{3}$ tan en moyenne, soit une dégradation spécifique de $23 \mathrm{t} / \mathrm{km}^{2} / \mathrm{an} \mathrm{com}$ parable à celle de l'Adour (18 $\mathrm{t} / \mathrm{km}^{2} / \mathrm{an}$; Snoussi, 1988) et du Girou $\left(20,1 \mathrm{t} / \mathrm{km}^{2} / \mathrm{an} ; \quad\right.$ Etchanchu, 1988), mais bien inférieure à celle de nombreuses autres rivières françaises: Garonne: 30t/ $\mathrm{km}^{2} / \mathrm{an}$ (Etchanchu, 1988); Moselle: $40 \mathrm{t} / \mathrm{km}^{2} / \mathrm{an}$ (Kattan, 1989). Ce tonnage est supérieur à celui de la Seine : 9,5 $\mathrm{t} / \mathrm{km}^{2} / a n$ (Cossa et al., 1994). Robinson (1977) donne une moyenne de $32 \mathrm{t} / \mathrm{km}^{2} / \mathrm{an}$ pour les rivières européennes. On peut rappeler ici qu'au niveau du globe, c'est dans les fleuves issus de la chaîne himalayenne que les tonnages les plus élevés sont observés (Ganges:

Tableau VIII. - Flux annuels et spécifiques des métaux particulaires dans l'Orne à May/Orne durant les cycles hydrologiques 1993-1994 et 1944-1995

Table VIII. - Annual and specific fluxes of the particulate metals in Orne at May/Orne for the hydrological cycles 1993-1994 and 1994-1995

références: " : Kattan. 1989; " "Cossa et al.. 1994; "** : Snoussi. 1988

Flux des métaux majeurs ("1 000T/an)

Flux des métaux traces $(\mathrm{Kg} / \mathrm{an})$

\begin{tabular}{|c|c|c|c|c|c|c|c|c|c|c|c|c|c|c|}
\hline & Si & Al & $\mathrm{Fe}$ & $\mathrm{Ca}$ & $\mathrm{Mg}$ & $\mathrm{Na}$ & K & $\pi$ & $\mathrm{Cd}$ & $\mathrm{Cu}$ & $\mathrm{Cr}$ & $\mathrm{Pb}$ & $\mathrm{Zn}$ & $\mathrm{Ni}$ \\
\hline & & 070 & 0 & 00 & 0 & 00 & 707 & 0.09 & . & 0 & & & & \\
\hline & & 2.78 & 2.02 & 0.89 & 0.42 & 0.35 & 0.70 & 0.15 & 161 & 3320 & 2840 & 2920. & 12100 & 2410 \\
\hline
\end{tabular}

Flux spécifiques en $\mathrm{Kg} / \mathrm{Km}^{2} / \mathrm{an}$

\begin{tabular}{|c|c|c|c|c|c|c|c|c|c|c|c|c|c|c|}
\hline Orne & 3965 & 885 & 633 & 287 & 150 & 123 & 231 & 48 & 0.04 & 0.97 & 0.85 & 0.81 & 3.47 & 0.71 \\
\hline Moselle" & 5640 & 1000 & 540 & 440 & 440 & 60 & 340 & 10.5 & - & 2.48 & 3.35 & - & 12.10 & 1.89 \\
\hline Seine ${ }^{* *}$ & - & - & - & - & - & - & - & - & 0.03 & 1.45 & - & 1.55 & 5.82 & - \\
\hline Adour ${ }^{* * *}$ & 7600 & 050 & 600 & 470 & 132 & 54 & 190 & 72 & - & 1.1 & 0.88 & 1.1 & 3 & 0.5 \\
\hline
\end{tabular}


$1128 \mathrm{t} / \mathrm{km}^{2} / \mathrm{an} ;$ Milliman et Meade, 1983). Le transport en suspension augmente avec l'altitude moyenne des bassins de drainage. Les flux spécifiques des métaux sont globalement comparables à ceux observés sur d'autres rivières françaises.

Pour calculer l'ablation mécanique dans le bassin, nous corrigeons ce tonnage des apports dus à l'érosion des berges et du lit de la rivière, érosion interne au cours d'eau, pour ne tenir compte que de l'érosion des versants. Ces apports sont calculés à partir des relations débit-concentration établies pour la période des basses eaux et qu'on extrapole aux hautes eaux. Le tonnage de sédiments apportés à la rivière par ruissellement superficiel serait de l'ordre de 48000 t/an, soit $76 \%$ du flux total de matériaux en suspension sont évacués en période de crue. Ce tonnage correspond à un transport spécifique de $17,7 \mathrm{t} / \mathrm{km}^{2}$ /an qui donne, pour une densité moyenne de 2,6 pour les roches superficielles, un abaissement du relief dans le bassin versant de l'Orne de $6,8 \mathrm{~mm} / 1000$ ans.

\section{CONCLUSION}

Les concentrations en matériel en suspension de l'Orne, relativement faibles $(0,2$ à $120 \mathrm{mg} / \mathrm{l})$, caractérisent un système peu turbide. Elles sont inférieures à $25 \mathrm{mg} / \mathrm{l}$ en période de basses eaux où le développement planctonique constitue la source prin- cipale des particules en suspension, et augmentent considérablement en hautes eaux ( $55 \mathrm{mg} / \mathrm{l}$ en moyenne) du fait essentiellement du lessivage des sols du à des averses à caractère brutal qui génèrent des crues dans des temps très courts. Les transports solides s'effectuant principalement lors des périodes de crue, les matériaux en suspension sont directement liés au ruissellement superficiel sur les versants: $76 \%$ de la charge solide étant exportée au cours de ces périodes dont la durée ne représente que $10 \%$ du temps annuel.

Les variations spatiales et saisonnières des concentrations des éléments métalliques dans les MES correspondent globalement à la lithologie des sous bassins drainés, et suivent l'évolution des débits. Ces caractéristiques ainsi que les faibles valeurs du facteur d'enrichissement, impliquent que la variation des teneurs métalliques est contrôlée par des processus surtout naturels, conditionnés par l'intensité des apports détritiques en provenance de l'érosion du bassin versant. Néanmoins, les teneurs en $\mathrm{Cd}$ accusent une notable influence anthropique dont lorigine n'est pas rigoureusement déterminée. Le transport de la plupart des éléments est lié en premier lieu aux minéraux argileux. $\mathrm{Cr}$ et $\mathrm{Cu}$ apparaissent également complexés à la matière organique. Une fraction importante du Ti est associée au quartz et notamment dans les fractions granulométriques les plus grossières. Les flux spécifiques des éléments ex- 
portés sont globalement comparables à ceux observés sur d'autres bassins français.

Cette étude a permis de mettre en évidence l'absence de pollution métallique dans ce bassin des régions tempérées industrielles qui sont soumises généralement à de fortes pollutions anthropiques. II peut servir, à notre sens, de référence pour des études similaires menées dans des bassins des régions tempérées industrielles. En outre, ce bassin constitue un atelier remarquable pour les études portant sur les processus d'érosion mécanique, de par la nature diversifiée du sous-sol, des formations superficielles et des écoulements.

\section{RÉFÉRENCES BIBLIOGRAPHIQUES}

Abdel-Hamid M.I., Shaaban-Dessouki S.A. et Skulberg O.M., 1992. Water quality of the river Nile in Egypt, 1: Physical and chemical characteristics. Arch. Hydrobiol. Suppl., 90 (3) : 283310.

Baisch P., 1994. Les oligo-éléments métalliques du système fluvio-lagunaire Dos Patos (Bresil). Flux et devenir. Thèse Doc., Univ. Bordeaux I, 229 pp.

Cossa D., Meybeck M., Idlafkih Z. et Bombled B., 1994. Étude pilote des apports en contaminants par la Seine. Rapport IFREMER-AESN-Ministere de l'Environnement, $89 \mathrm{p}$ et annexes.

Dupont J.P., Lafite R., Huault M.F., Hommeril P. et Meyer R., 1994. Continen$\mathrm{tal} /$ marine ratio changes in suspended and settled matter across a macrotidal estury (the Seine estuary, northwestern France). Marine Geology, 120: 27-40.

Etchanchu D., 1988. Géochimie des eaux du bassin de la Garonne. Transferts de matières dissoutes et particulaires vers l'Océan Atlantique. Thèse Doc., Université Paul Sabatier, Toulouse, $178 \mathrm{pp}$.

Horovitz A.J., Rinella F.A., Lamothe P., Miller T.L., Edwards T.K., Roche R.L. et Ricket D.A., 1990. Variations in suspended sediment and associated trace element concentrations in selected riverine cross sections. Environ. Sci. Technol., 24 : 1313-1320.

Idlafkih Z., Cossa D. et Meybeck M., 1995. Comportements des contaminants en trace dissous et particulaires (AS, Cd, $\mathrm{Cu}, \mathrm{Hg}, \mathrm{Pb}, \mathrm{Zn}$ ) dans la Seine. Hydroécol. Appl., 7 (1-2) : 127-150.

Johnson C.A., 1986. The regulation of trace element concentrations in river and estuarine waters contaminated with acid mine drainage : the adsorption of $\mathrm{Cu}$ and $\mathrm{Zn}$ on amorphous $\mathrm{Fe}$ oxyhydroxides. Geochim. Cosmochim. Acta, $50: 2433-2438$.

Kattan Z., 1989. Géochimie et hydrologie des eaux fluviales des bassins de la Moselle et de la Mossig. Transport dissous et particulaires. Cycles biogéochimiques des éléments. Thèse Doc., Université Louis Pasteur, Strasbourg, $218 \mathrm{pp}$.

Lafite R., 1995. Microgranulométrie des suspensions marines par compteur de particules Coulter. Spectra Analyse, $183: 35-38$.

Martin J.M. et Meybeck M., 1979. Elemental mass-balance of material carried by major world rivers. Mar. Chem., 7 : 173-206.

Martin J.M. et Whitfield M., 1983. The significance of the river input of chemical elements to the Ocean. In: Trace metals in sea water. New York, Plenum Press : 265-296. 
Meybeck M., 1984. Les fleuves et le cycle géochimique des éléments. Thèse de Doc. d'Etat, Univ. P. \&M. Curie, Paris VI, $437 \mathrm{pp}$.

Milliman J.D. et Meade R.H., 1983. Worldwide delivery of river sediment to the oceans. J. of Geol., 91: 1-21.

Nasseh A. Texier H., Lacroix M. et Verague J., 1996. Variabilité saisonnière des flux géochimiques dans un petit bassin versant tempéré: exemple de l'Orne (Calvados-France). Geomorphologie : Relief, Processus, Environnement, $3:$ 57-92.

Nirel P., 1987. Evolution de la distribution particulaire des éléments chimiques en milieu estuarien. Thèse Doc., Univ. Paris VII, 187 pp.

Ouddane B., Fischer J.C., et Wartel M., 1992. Evaluation statistique de la répartition des métaux en traces $\mathrm{Cd}, \mathrm{Pb}$, $\mathrm{Cu}, \mathrm{Zn}$ et $\mathrm{Mn}$ dans la Seine et son estuaire. Oceanol. Acta, 15 (4) : 347354.

Probst J.L., Nkounkou R.R., Krempp G., Bricquet J.P., Thiébaux J.P. et Olivry J.C., 1992. Dissolved major elements exported by the Congo and the Ubangi rivers during the period 1987-1989. Journal of Hydrology, 135 : 237-257.

Robinson A.R., 1977. Relationship between soil erosion and sediment delivery. Proc. Paris Symp., IAHS Publ., 122: 159-167.

Shankar R. et Manjunatha B.R., 1994. Elemental composition and particulate metal fluxes from Netravati and Gupur rivers to the Coastal Arabian Sea. J. Geol. Soc. India, 43 : 255-265.

Sinawi G. et Walling D.E., 1996. The characteristics of composite suspended sediment particles transported during storm events in the river Exe, Devon, U.K. Geomorphologie : Relief, Processus, Environnement, 2 : 29-40.

Snoussi M., 1988. Nature, estimation et comparaison des flux de matières issus des bassins versants de l'Adour (France), du Sebou, de l'Oum-er-Rbia et du Souss (Maroc). Mémoire de l'Institut de Géologie du Bassin d'Aquitaine. $n^{\circ} 22,459 \mathrm{pp}$.

Thomas R.L. et Meybeck M., 1992. The use of particulate material. In: Water quality and assessments. Chapman D. (eds). London, Chapman \& Hall : 121170.

Van Der Weijden C. $\mathrm{H}$. et Middelburg G.J.J., 1989. Hydrogeochemistry of the river Rhine, long term and seasonal variability, elemental budgets, base levels and pollution. Wat. Res., 23, 10 : 1247-1266.

Weiss A., Bertine K.K., Koide M. et Goldberg E.D., 1991. Scavenging of particulate matter in coastal waters. Mar. Chem., 32 (2-4) : 171-175.

Windom H., Gyrd J. et Smith R. Jr., 1991. Trace metal-nutrient relationships in estuaries. Mar. Chemistry, 32 : 177194.

Zhang J., Huang W.W. et Wang J.H., 1994. Trace-metal chemistry of the Huanghe, China. Examination of the data from in situ measurments and laboratory approach. Chemical Geology, $114: 83-94$

Zhang J., Huang W.W., Letolle R. et Jusserand C., 1995. Major element chemistry of the Huanghe (Yellow river), China. Weathering processes and chemical fluxes. J. Hydrol. Amst., 168 $(1-4): 173-203$. 